KODAI MATH. SEM. REP.

19 (1967), 1-27

\title{
ALMOST COMPLEX STRUCTURES INDUCED IN TANGENT BUNDLES
}

\author{
By Kentaro Yano and Shigeru Ishihara
}

Introduction. The differential geometry of tangent bundles of Riemannian manifolds has been studied by Sasaki [5] ${ }^{1)}$ and the theory of affine connections in tangent bundles of manifolds with affine connection by Ledger and one of the present authors [3], [15].

Kobayashi and one of the present authors [13], [14] recently studied prolongation of tensor fields and connections to tangent bundles. They first developed the general theory of prolongation of tensor fields and affine connections to tangent bundles and then studied affine transformations in tangent bundles.

The main purpose of the present paper is to study the prolongation of the socalled $f$-structure to tangent bundles and especially that of almost contact structure in the light of the above mentioned papers by Kobayashi and one of the present authors.

In Sections 1 and 2, we recall some results stated in [13] and [14] which are indispensable in the subsequent sections.

In Section 3, we study the so-called $f$-structure and its prolongation to the tangent bundle. To study the properties of the so-called $f$-structure, we introduce a frame closely related to the $f$-structure. We study these in Section 4 .

The last section is devoted to the study of prolongation of the so-called almost contact structure which is an $f$-structure. The results obtained in this section are closely related to those obtained recently by Tanno [9], [10].

\section{Contents}

$\S 1$. Lifts of tensor fields.

$\S 2$. Almost complex structures.

$\S 3$. $f$-structures.

$\S 4$. Frames and Lie groups.

$\S 5$. Almost contact structures and framed $f$-structures.

\section{$\S 1$. Lifts of tensor fields.}

We first recall definitions and properties of the vertical lift and the complete

Received April 2, 1966.

1) The number in brackets refers to the References at the end of the paper. 
lift of a tensor field to tangent bundle for the later use (Yano and Kobayashi [13], [14]). Let $V$ be an $n$-dimensional differentiable manifold ${ }^{2)}$, and $T(V)$ its tangent bundle, the projection being denoted by $\pi: T(V) \rightarrow V$. For a differentiable function $\phi$ in $V$, the function $\pi^{*} \phi$ induced from $\phi$ in $T(V)$ is denoted by

$$
\phi^{V}=\pi^{*} \phi
$$

and is called the vertical lift of the function $\phi$. Any 1-form $\omega$ given in $V$ is, in a natural way, regarded as a function in $T(V)$, which will be denoted by $\iota \omega$. If we are given a vector field $X$ in $V$, then we define a vector field $X^{V}$ in $T(V)$ by

$$
X^{v}(\iota \omega)=(\omega(X))^{v},
$$

$\omega$ being an arbitrary 1 -form in $V .^{3)}$ The vector field $X^{v}$ thus defined is called the vertical lift of the vector field $X$.

We define the vertical lifts of 1 -forms $d \phi$ and $\phi d \phi$ by

$$
(d \phi)^{V}=d\left(\phi^{V}\right) \quad \text { and } \quad(\phi d \phi)^{V}=\phi^{V}(d \phi)^{V}
$$

respectively, $\phi$ and $\phi$ being arbitrary functions in $V$, and the vertical lift $\omega^{V}$ of an arbitrary 1 -form $\omega$ given in $V$ by $^{4)}$

$$
\omega^{V}=\left(\omega_{i}\right)^{V}\left(d x^{i}\right)^{V}
$$

in each open set $\pi^{-1}(U)$, where $U$ is a coordinate neighborhood with local coordinates $\left(x^{h}\right)$ in $V$ and $\omega$ is given by $\omega=\omega_{i} d x^{2}$ in $U$. It is easily verified that the vertical lift $\omega^{V}$ of the 1 -form $\omega$ defined by (1.4) in each $\pi^{-1}(U)$ is a global 1 -form in $T(V)$.

When there is given a function $\phi$ in $V$, we put

$$
\phi^{C}=\iota(d \phi)
$$

in $T(V)$, and call the function $\phi^{C}$ thus defined in $T(V)$ the complete lift of the function $\phi$. For a vector field $X$ given in $V$, we define a vector field $X^{c}$ in $T(V)$ by

$$
X^{C} \phi^{C}=(X \phi)^{C},
$$

$\phi$ being an arbitrary function in $V$, and call $X^{c}$ the complete lift of the vector field $X^{.5}$

2) The manifolds, tensor fields and mappings we discuss are assumed to be differentiable and of class $C^{\infty}$. The manifolds are assumed to be connected.

3) The 1-form $\omega$ being arbitrary in $V$, the function $\iota \omega$ generates the ring of all functions in $T(V)$, so $X^{v}(\iota \omega)=(\omega(X))^{v}$ defines a vector field $X^{v}$ in $T(V)$ (cf. [13]).

4) The indices $h, i, i, k, l$ run over the range $\{1,2, \cdots, n\}$.

5) The function $\phi$ being arbitrary in $V$, the function $\phi^{C}$ generates the ring of all functions in $T(V)$, so $X^{C} \phi^{C}=(X \phi)^{C}$ defines a vector field $X^{C}$ in $T(V)$ (cf. [13]). 
Given a 1 -form $\omega$ in $V$, we define a 1 -form $\omega^{C}$ in $T(V)$ by

$$
\omega^{C}\left(X^{C}\right)=(\omega(X))^{C},
$$

$X$ being an arbitrary vector field in $V^{6}{ }^{6}$ The 1 -form $\omega^{C}$ thus defined in $T(V)$ is called the complete lift of the 1-form $\omega$. Let $F$ be a tensor field of type (1.1) in $V$. Then we define in $T(V)$ its vertical lift $F^{V}$ and its complete lift $F^{c}$ by

$$
F^{v} X^{c}=(F X)^{V} \quad \text { and } \quad F^{c} X^{C}=(F X)^{C}
$$

respectively, $X$ being an arbitrary vector field given in $V$. For a tensor field $S$ of type $(1,2)$ given in $V$, we define in $T(V)$ its vertical lift $S^{V}$ and its complete lift $S^{C}$ respectively by

$$
S^{v}\left(X^{c}, Y^{c}\right)=(S(X, Y))^{V} \quad \text { and } \quad S^{c}\left(X^{c}, Y^{c}\right)=(S(X, Y))^{c},
$$

$X$ and $Y$ being arbitrary vector fields in $V$.

We shall now give local representations of the lifts. Let $\left(U,\left(x^{h}\right)\right)$ be a coordinate neighborhood of the differentiable manifold $V$, where $\left(x^{h}\right)$ is a system of local coordinates defined in $U$. Let $\left(y^{h}\right)$ be the system of cartesian coordinates in each tangent space $T_{\mathrm{P}}(V)$ of $V$ at $\mathrm{P}$ with respect to the natural frame $\partial / \partial x^{h}$, where $\mathrm{P}$ is an arbitrary point belonging to $U$. Then, in the open set $\pi^{-1}(U)$ of $T(U)$ we can introduce local coordinates $\left(x^{h}, y^{h}\right)$, which are called the coordinates induced in $\pi^{-1}(U)$ from $\left(x^{h}\right)$ or simply induced coordinates in $\pi^{-1}(U)$. Let there be given a function $\phi\left(x^{k}\right)$ in $U$. Then its vertical lift $\phi^{V}$ and its complete lift $\phi^{C}$ are respectively represented by

$$
\phi^{V}: \phi\left(x^{k}\right) \quad \text { and } \quad \phi^{C}: \partial \phi\left(x^{k}\right)
$$

in $\pi^{-1}(U)$ with respect to the induced coordinates $\left(x^{h}, y^{h}\right)$, where the symbol $\partial$ denotes the operator

$$
\partial=y^{2} \frac{\partial}{\partial x^{2}}
$$

If a vector field $X$ has components $X^{h}$ in $U$, then its vertical lift $X^{V}$ and its complete lift $X^{C}$ have respectively components of the form

$$
X^{v}:\left(\begin{array}{c}
0 \\
X^{h}
\end{array}\right) \text { and } X^{c}:\left(\begin{array}{c}
X^{h} \\
\partial X^{h}
\end{array}\right)
$$

in $\pi^{-1}(U)$ with respect to the induced coordinates $\left(x^{h}, y^{h}\right)$.

6) The vector field $X$ being arbitrary in $V$, the vector field $X^{C}$ in $T(V)$ spans the set of all vector fields in $T(V)$, so a 1 -form $\omega^{C}$, tensor fields $F^{V}, F^{C}$ of type (1.1) and tensor fields $S^{V}, S^{C}$ of type (1.2) are completely determined by (1.7), (1.8) and (1.9) respectively (cf. [13]). 
If a 1 -form $\omega$ has components $\omega_{i}$ in $U$, i.e. if $\omega=\omega_{i} d x^{i}$ in $U$, then its vertical lift $\omega^{V}$ and its complete lift $\omega^{C}$ have respectively components of the form

$$
\omega^{V}:\left(\omega_{i}, 0\right) \quad \text { and } \quad \omega^{C}:\left(\partial \omega_{i}, \omega_{i}\right),
$$

i.e.

$$
\omega^{V}=\omega_{i} d x^{i} \quad \text { and } \quad \omega^{C}=\left(\partial \omega_{i}\right) d x^{i}+\omega_{i} d y^{2}
$$

in $\pi^{-1}(U)$ with respect to the induced coordinates $\left(x^{h}, y^{h}\right)$.

If a tensor field $F$ of type $(1,1)$ has components $F_{i}^{h}$ in $U$, then its vertical lift $F^{V}$ and its complete lift $F^{C}$ have respectively components of the form

$$
F^{V}:\left(\begin{array}{cc}
0 & 0 \\
F_{i}^{h} & 0
\end{array}\right) \text { and } F^{C}:\left(\begin{array}{cc}
F_{i}^{h} & 0 \\
\partial F_{i}^{h} & F_{i}^{h}
\end{array}\right)
$$

in $\pi^{-1}(U)$ with respect to the induced coordinates $\left(x^{h}, y^{h}\right)$. Thus the complete lift of the identity tensor field of $V$ is the identity tensor field in $T(V)$.

Taking account of the definitions of lifts or of their local representations (1. 10), (1.11), (1.12) and (1.13), we obtain the following formulas:

$$
\begin{aligned}
& (\phi \phi)^{V}=\phi^{V} \phi^{V}, \quad(\phi \phi)^{C}=\phi^{V} \phi^{C}+\phi^{C} \phi^{V}, \\
& (\phi X)^{v}=\phi^{v} X^{v}, \quad(\phi X)^{c}=\phi^{v} X^{c}+\phi^{c} X^{v}, \\
& (\phi \omega)^{V}=\phi^{V} \omega^{V}, \quad(\phi \omega)^{C}=\phi^{V} \omega^{C}+\phi^{C} \omega^{V}, \\
& (X \otimes \omega)^{V}=X^{V} \otimes \omega^{V}, \quad(X \otimes \omega)^{C}=X^{V} \otimes \omega^{C}+X^{C} \otimes \omega^{V}
\end{aligned}
$$

for any functions $\phi, \phi$, any vector field $X$ and any 1-form $\omega$ given in $V$;

$$
\begin{array}{ll}
X^{v} \phi^{V}=0, & X^{v} \phi^{C}=(X \phi)^{V}, \\
X^{C} \phi^{V}=(X \phi)^{V}, & X^{c} \phi^{C}=(X \phi)^{C}
\end{array}
$$

for any function $\phi$ and any vector field $X$ given in $V$;

$$
\begin{array}{ll}
\omega^{V}\left(X^{V}\right)=0, & \omega^{V}\left(X^{C}\right)=(\omega(X))^{V}, \\
\omega^{C}\left(X^{V}\right)=(\omega(X))^{V}, & \omega^{C}\left(X^{C}\right)=(\omega(X))^{C}
\end{array}
$$

for any vector field $X$ and any 1 -form $\omega$ given in $V$;

$$
\left[X^{v}, Y^{v}\right]=0 \text {, }
$$$$
\left[X^{v}, Y^{c}\right]=\left[X^{c}, Y^{v}\right]=[X, Y]^{v} \text {, }
$$$$
\left[X^{c}, Y^{c}\right]=[X, Y]^{c}
$$ 
for any two vector fields $X$ and $Y$ given in $V$, and

$$
\begin{array}{ll}
F^{v} X^{v}=0, & F^{c} X^{v}=(F X)^{v}, \\
F^{v} X^{C}=(F X)^{v}, & F^{c} X^{c}=(F X)^{C}
\end{array}
$$

for any vector field $X$ and any tensor field $F$ of type $(1,1)$ given in $V$.

Let there be given two tensor fields $F$ and $K$ of type $(1,1)$ in $V$. Then we find directly from the definition (1.8) of complete lifts

$$
F^{c} K^{c}=(F K)^{c} \text {. }
$$

Thus, if there are given a tensor field $F$ of type $(1,1)$ and a polynomial $\Phi(t)$ of a variable $t$, then we get from (1.19)

$$
(\Phi(F))^{C}=\Phi\left(F^{C}\right)
$$

for example,

$$
\left(F^{2}+I\right)^{C}=\left(F^{C}\right)^{2}+I, \quad\left(F^{3}+F\right)^{C}=\left(F^{C}\right)^{3}+F^{C},
$$

where $I$ denotes the identity tensor field of type $(1,1)$ in the corresponding manifold $V$ or $T(V)$.

For a vector field $\tilde{X}$ and a tensor field $\tilde{F}$ of type $(1,1)$ given in $T(V)$, the Lie derivative $\underset{\tilde{X}}{\mathcal{L}} \tilde{F}$ of $\tilde{F}$ with respect to $\tilde{X}$ is defined by

$$
(\underset{\tilde{X}}{\mathcal{F}} \tilde{F} \tilde{Y}=[\tilde{X}, \tilde{F} \tilde{Y}]-\tilde{F}[\tilde{X}, \tilde{Y}] \text {, }
$$

$\tilde{Y}$ being an arbitrary vector field in $T(V)$. Thus, putting $\tilde{X}=X^{c}, \tilde{Y}=Y^{c}$ and $\tilde{F}=F^{c}$, we obtain

$$
\begin{aligned}
\underset{X^{C}}{\left(\mathcal{L} F^{C}\right) Y^{C}} & =\left[X^{C}, F^{C} Y^{C}\right]-F^{C}\left[X^{C}, Y^{C}\right] \\
& =([X, F Y]-F[X, Y])^{C} \\
& =(\underset{X}{\mathcal{L} F})^{C} Y^{C}
\end{aligned}
$$

by means of (1.17) and (1.18), where $X$ and $Y$ are vector fields in $V$ and $F$ is a tensor field of type $(1,1)$ in $V$. The vector field $Y$ being arbitrary, we find from the equation above the following formula:

$$
\underset{X^{C}}{\mathcal{L} F^{C}}=\underset{X}{(\mathcal{L} F)^{C}}
$$

for any vector field $X$ and any tensor field $F$ of type $(1,1)$ in $V$. Similarly, we obtain the following formula:

$$
\underset{X V}{\mathcal{L} F} F^{C}=(\underset{X}{\mathcal{L} F})^{V}
$$

for any vector field $X$ and any tensor field $F$ of type $(1,1)$ in $V$. 
Let there be given a tensor field $F$ of type $(1,1)$ in $V$. Then its Nijenhuis tensor $N$ is by definition

$$
N(X, Y)=[F X, F Y]-F[F X, Y]-F[X, F Y]+F^{2}[X, Y],
$$

$X$ and $Y$ being arbitrary vector fields in $V$. Taking the complete lifts of both sides of (1.25), and taking account of (1.9), (1.17) and (1.18), we obtain

$$
N^{C}\left(X^{c}, Y^{c}\right)=\left[F^{c} X^{c}, F^{c} Y^{c}\right]-F^{C}\left[F^{c} X^{c}, Y^{c}\right]-F^{C}\left[X^{c}, F^{C} Y^{C}\right]+\left(F^{C}\right)^{2}\left[X^{C}, Y^{c}\right] .
$$

The right hand side of the equation above is nothing but the Nijenhuis tensor $\tilde{N}\left(X^{c}, Y^{c}\right)$ of the complete lift $F^{c}$ of $F$. Thus we have

$$
N^{C}=\tilde{N}
$$

where $\tilde{N}$ denotes the Nijenhuis tensor of $F^{C}$.

It is easily seen that a tensor field $K$ (vector field, 1-form, tensor field of higher degree), which is not a function, vanishes identically in $V$ if and only if its vertical lift $K^{V}$ or its complete lift $K^{c}$ does so in $T(V)$. A function $\phi$ vanishes identically in $V$ if and only if its vertical lift $\phi^{V}$ does so in $T(V)$. A function $\phi$ is a constant in $V$ if and only if its complete lift $\phi^{C}$ vanishes identically in $T(V)$. Let $F$ be a tensor field of type $(1,1)$ in $V$. Then its vertical lift $F^{V}$ is of rank $r$ and its complete lift $F^{C}$ is of rank $2 r$ if and only if $F$ is of rank $r$.

Let there be given a distribution $M$ in $V$ and suppose that $M$ is determined by a projection tensor $m$, i.e. that $m$ is a tensor field of type $(1,1)$ in $V$ such that $m^{2}=m$ and $m T(V)=M$. The complete lift $m^{C}$ of the tensor field $m$ is a projection tensor in $T(V)$, i.e.

$$
\left(m^{C}\right)^{2}=m^{C}
$$

by virtue of (1.20). The distribution $M^{C}$ in $T(V)$ determined by the projection tensor $m^{C}$ is called the complete lift of the distribution $M$. It is easily verified by means of (1.18) that the complete lift $M^{C}$ is spanned by all vector fields of the type $X^{v}$ and all vector fields of the type $X^{c}, X$ being an arbitrary vector field belonging to the distribution $M$. The distribution $M$ is integrable if and only if we have

$$
l[m X, m Y]=0
$$

for any vector fields $X$ and $Y$ in $V$, where $l=I-m$. Taking the complete lifts of the both sides in (1.27), we get

$$
l^{C}\left[m^{C} X^{C}, m^{C} Y^{C}\right]=0
$$

for any vector fields $X$ and $Y$ in $V$. The two conditions (1.27) and (1.28) being equivalent to each other, we have 
Proposition 1.1. The complete lift $M^{C}$ of a distribution $M$ given in $V$ is integrable in $T(V)$ if and only if $M$ is integrable in $V$.

Let there be given a differentiable transformation

$$
\mu: \quad V \rightarrow V
$$

and denote simply by

$$
\mu: \quad T(V) \rightarrow T(V)
$$

the differential of the transformation $\mu: V \rightarrow V$. For a 1-form $\omega$ in $V$, we define a 1-form $\mu \omega$ by

$$
(\mu \omega)(X)=\omega(\mu X),
$$

$X$ being an arbitrary vector field in $V$. If the differential of the transformation $\mu: T(V) \rightarrow T(V)$ is denoted by

$$
\mu^{c}: \quad T(T(V)) \rightarrow T(T(V)),
$$

then we get the following formulas:

$$
(\mu X)^{V}=\mu^{C} X^{v}, \quad(\mu X)^{C}=\mu^{C} X^{c},
$$

$$
(\mu \omega)^{V}=\mu^{C} \omega^{V}, \quad(\mu \omega)^{C}=\mu^{C} \omega^{c}
$$

for any vector field $X$ and any 1-form $\omega$ given in $V$. If, for a tensor field $F$ of type $(1,1)$ given in $V$, we define a tensor field $\mu F$ by

$$
(\mu F)(X)=\mu^{-1}(F(\mu X)),
$$

then we obtain

$$
(\mu F)^{V}=\mu^{C} F^{V}, \quad(\mu F)^{C}=\mu^{C} F^{C} .
$$

The tensor field $\mu F$ is that induced from the given $F$ by the transformation $\mu: V \rightarrow V$.

\section{§ 2. Almost complex structures.}

Let $F$ be a tensor field of type $(1,1)$ in a differentiable manifold $V$. Then we see, taking account of (1.21), that

$$
\left(F^{C}\right)^{2}+I=0
$$

if and only if 


$$
F^{2}+I=0
$$

Thus we have

Proposition 2.1. The complete lift $F^{G}$ of a tensor field $F$ of type $(1,1)$ given in $V$ is an almost complex structure in $T(V)$ if and only if so is $F$ in $V$. (Satō [8], Yano and Kobayashi [13])

Taking account of (1.26), we have

Proposition 2.2. The complete lift $F^{C}$ of an almost complex structure $F$ given in $V$ is complex analytic in $T(V)$ if and only if $F$ is complex analytic in $V$. (Yano and Kobayashi [13])

The equations (1.23) and (1.24) imply

Proposition 2.3. A vector field $X$ is almost analytic in an almost complex space $V$ with an almost complex structure $F$ if and only if the vertical lift $X^{V}$ or the complete lift $X^{C}$ is almost analytic with respect to the almost complex structure $F^{C}$ in $T(V)$. (Satō [8], Yano and Kobayashi [13])

We have from (1.30)

Proposition 2.4. In an almost complex space $V$ with an almost complex structure $F$, a transformation $\mu: V \rightarrow V$ preserves the structure $F$ if and only if its differential map $\mu: T(V) \rightarrow T(V)$ preserves $F^{c}$.

\section{$\S 3$. $f$-structures.}

Let there be given, in an $n$-dimensional differentiable manifold $V$, a non-null tensor field $f$ of type $(1,1)$ satisfying

$$
f^{3}+f=0 .
$$

We call such a structure $f$ an $f$-structure of rank $r$, when the rank of $f$ is constant everywhere in $V$ and is equal to $r, r$ being necessarily even (Yano [11], [12]). If we put

$$
l=-f^{2}, \quad m=f^{2}+I
$$

then we have

$$
\begin{gathered}
l+m=I, \quad l^{2}=l, \quad m^{2}=m, \quad l m=m l=0, \\
f l=l f=f, \quad f m=m f=0 .
\end{gathered}
$$

These equations show that there exist in $V$ two complementary distributions $L$ 
and $M$ corresponding to the projection tensors $l$ and $m$ respectively. When the rank of $f$ is $r, L$ is $r$-dimensional and $M(n-r)$-dimensional.

We have obtained in [2] the following integrability conditions:

(A) A necessary and sufficient condition for the distribution $M$ to be integrable is that

$$
N(m X, m Y)=0
$$

for any vector fields $X$ and $Y, N$ denoting the Nijenhuis tensor of the $f$-structure $f$.

(B) A necessary and sufficient condition for the distribution $L$ to be integrable is that

$$
m N(X, Y)=0
$$

for any vector fields $X$ and $Y$ in $V$.

When the distribution $L$ is integrable, the $f$-structure $f$ operates as an almost complex structure $f^{\prime}$ in each integral manifold of $L$. When the distribution $L$ is integrable and the induced almost complex structure $f^{\prime}$ is complex analytic in each integral manifold of $L$, we say that the $f$-structure $f$ is partially integrable.

(C) A necessary and sufficient condition for an $f$-structure $f$ to be partially integrable is that

$$
N(l X, l Y)=0
$$

for any vector fields $X$ and $Y$ in $V$.

We suppose now that there exist, in each coordinate neighborhood of $V$, local coordinates, with respect to which the $f$-structure $f$ has constant components:

$$
f=\left(\begin{array}{ccc}
0 & -E_{m} & 0 \\
E_{m} & 0 & 0 \\
0 & 0 & 0
\end{array}\right),
$$

where $r=2 m$ is the rank of $f$ and $E_{m}$ denotes the unit $m \times m$ matrix. If this is the case, we say that the $f$-structure $f$ is integrable. is that

(D) A necessary and sufficient condition for an $f$-structure $f$ to be integrable

$$
N(X, Y)=0
$$

for any vector fields $X$ and $Y$ in $V$.

Let $F$ be a tensor field of type $(1,1)$ in a differentiable manifold $V$. Then we see, taking account of (1.21), that the equation 


$$
F^{3}+F=0
$$

is equivalent to the equation

$$
\left(F^{C}\right)^{3}+F^{C}=0 .
$$

The rank of $F^{C}$ is $2 r$ if and only if the rank of $F$ is $r$. Thus we have

Proposition 3.1. The complete lift $F^{C}$ of a tensor field $F$ of type $(1,1)$ given in $V$ is an $f$-structure in $T(V)$ if and only if so is $F$ in $V$. When $F$ is of rank $r$ in $V, F^{c}$ is of rank $2 r$ in $T(V)$. (Yano and Kobayashi [13])

Let $f$ be an $f$-structure of rank $r$ in $V$. Then the complete lifts $l^{c}$ and $m^{C}$ are complementary projection tensors in $T(V)$, where $l$ and $m$ are defined by (3.2). Thus there exist in $T(V)$ two complementary distributions $L^{C}$ and $M^{C}$ determined by $l^{C}$ and $m^{C}$ respectively. The distributions $L^{C}$ and $M^{C}$ are respectively the complete lifts of the distributions $L$ and $M$. Denote by $N$ and $\tilde{N}$ the Nijenhuis tensors of $f$ and $f^{C}$ respectively. Then, by means of (1.9), (1.18) and (1.26), the conditions (3.4), (3.5), (3.6) and (3.7) are respectively equivalent to the following conditions:

$$
\begin{aligned}
\tilde{N}\left(m^{c} Y^{c}, m^{c} Y^{c}\right) & =0, \\
m^{c} \tilde{N}\left(X^{c}, Y^{c}\right) & =0, \\
\tilde{N}\left(l^{c} X^{c}, l^{c} Y^{c}\right) & =0, \\
\tilde{N}\left(X^{c}, Y^{c}\right) & =0,
\end{aligned}
$$

$X$ and $Y$ being arbitrary vector fields in $V$. Therefore we obtain

Proposition 3.2. The complete lift $f^{C}$ of an $f$-structure $f$ given in $V$ satisfies one of integrability conditions (A), (B), (C) and (D) in $T(V)$ if and only if the given $f$-structure $f$ satisfies the corresponding integrability condition in $V$.

Let there be given an $f$-structure $f$ of $\operatorname{rank} r$ in $V$ and assume that there exist $s(=n-r)$ vector fields $\xi_{1}, \xi_{2}, \cdots, \xi_{s}$ spanning the distribution $M$ at each point of $V$. Then the set $\left(f, \xi_{x}\right)$ of an $f$-structure $f$ and such vector fields $\xi_{x}$ is called a framed $f$-structure ${ }^{7)}$ (cf. Nakagawa [4]). It was proved in [1] and [4] that there exists a natural almost complex structure $F$ in the product space $V \times R^{s}$ of the given manifold $V$ and the space $R^{s}$ of $s$ real variables if there is given a framed $f$-structure $\left(f, \xi_{x}\right)$ in $V$. When the almost complex structure $F$ is complex analytic in $V \times R^{s}$, we say that the given framed $f$-structure $\left(f, \xi_{x}\right)$ is normal (c.f. Ishihara [1], Nakagawa [4]). It is proved in [1] and [4] that a framed $f$-structure $\left(f, \xi_{x}\right)$ is normal if and only if we have

7) The indices $x, y, z, u, v$ run over the range $\{1,2, \cdots, s\}$. 


$$
S(X, Y)=0
$$

for any vector fields $X$ and $Y$ in $V, S$ being a tensor field of type $(1,1)$ defined in $V$ by the equation

$$
S(X, Y)=N(X, Y)+\left(X \eta^{x}(Y)-Y \eta^{x}(X)-\eta^{x}([X, Y])\right) \xi_{x}
$$

for any vector fields $X$ and $Y$ in $V$, where $N$ is the Nijenhuis tensor of $f$ and $\eta^{1}, \eta^{2}, \cdots, \eta^{s}$ are $s$ covector fields in $V$ satisfying

$$
\eta^{x}(f X)=0, \quad \eta^{x}\left(\xi_{y}\right)=\delta_{y}^{x}
$$

for any vector field $X$ in $V$. The tensor field $S$ thus defined is uniquely determined up to a linear transformation of $\xi_{x}$ with constant coefficients.

Let $\left(f, \xi_{x}\right)$ be a framed $f$-structure in $V$. Then the complete lifts $f^{C}, \xi_{x}{ }^{C}$ and the vertical lifts $\xi_{x}{ }^{V}$ form a framed $f$-structure $\left(f^{C}, \xi_{x}{ }^{V}, \xi_{x}{ }^{C}\right)$ in $T(V)$. The tensor field $\widetilde{S}$ which is constructed from $\left(f^{C}, \xi_{x} V, \xi_{x}^{C}\right)$ and corresponds to the tensor field $S$ defined by (3.9) is given by

$$
\begin{aligned}
\tilde{S}(\tilde{X}, \tilde{Y})=\tilde{N}(\tilde{X}, \tilde{Y}) & +\left(\tilde{X} \eta^{x_{V}}(\tilde{Y})-\tilde{Y} \eta^{x_{V}}(\tilde{X})\right)-\eta^{x_{V}}([\tilde{X}, \tilde{Y}]) \xi_{x}{ }^{C} \\
& +\left(\tilde{X} \eta^{x_{C}}(\tilde{Y})-\tilde{Y} \eta^{x_{C}}(\tilde{X})-\eta^{x_{C}}([\tilde{X}, \tilde{Y}])\right) \xi_{x}{ }^{V}
\end{aligned}
$$

for any vector fields $\tilde{X}$ and $\tilde{Y}$ in $T(V), \eta^{x}$ being the $s$ covector fields satisfying (3.10) in $V$, where $\tilde{N}$ is the Nijenhuis tensor of $f^{C}$. Taking account of (1.6), (1.9), (1.14), (1.15), (1.16), (1.17), (1.18) and (1.26), we find from (3.9) and (3.11) the equation $\widetilde{S}\left(X^{c}, Y^{c}\right)=S^{C}\left(X^{c}, Y^{C}\right)$ for any vector fields $X$ and $Y$ in $V$, i.e.

$$
\widetilde{S}=S^{C} \text {. }
$$

Thus we have

Proposition 3. 3. Let there be given a framed $f$-structure $\left(f, \xi_{x}\right)$ in $V$. Then $\left(f^{C}, \xi_{x^{V}}, \xi_{x^{C}}\right)$ is a framed $f$-structure in $T(V)$. The framed $f$-structure $\left(f^{C}, \xi_{x}{ }^{V}, \xi_{x}{ }^{C}\right)$ is normal in $T(V)$ if and only if the framed $f$-structure $\left(f, \xi_{x}\right)$ is normal in $V$.

The framed $f$-structure $\left(f^{C}, \xi_{x} V, \xi_{x}{ }^{C}\right)$ in Proposition 3. 3 is called the complete lift of the given framed $f$-structure $\left(f, \xi_{x}\right)$.

\section{$\S 4$. Frames and Lie groups.}

Let there be given, in an $n$-dimensional differentiable manifold $V, s$ vector fields $\xi_{1}, \xi_{2}, \cdots, \xi_{s}$ linearly independent at each point of $V$. Then there exists an $(n-s)$-dimensional distribution $M$ which is complementary to the distribution $L$ spanned by $s$ given vector fields $\xi_{x}$. Assuming such a distribution $M$ to be fixed, 
we call the set $\left(\xi_{x}, M\right)$ of $s$ vector fields $\xi_{x}$ and such a distribution $M$ an $s$-frame in $V$.

When an $s$-frame $\left(\xi_{x}, M\right)$ is given in $V$, there exist $s$ covector fields $\eta^{1}, \eta^{2}, \cdots, \eta^{s}$ satisfying

$$
\eta^{x}\left(\xi_{y}\right)=\delta_{y}^{x}, \quad \eta^{x}(X)=0
$$

for any vector field $X$ belonging to the distribution $M$. Defining a tensor field $\tilde{J}$ of type $(1,1)$ in $T(V)$ by

$$
\widetilde{J}=\xi_{x} V \otimes \eta^{x \cdot}-\xi_{x}^{C} \otimes \eta^{x C}
$$

which is of rank $2 s$ everywhere in $T(V)$, and taking account of (1.16), we find as a consequence of (4.1)

$$
\widetilde{J}^{2}=-\left(\xi_{x} \otimes \eta^{x}\right)^{C}
$$

and hence

$$
\widetilde{J}^{3}+\widetilde{J}=0
$$

and consequently, we see that the tensor field $\tilde{J}$ thus defined and the vector fields $\xi_{x} V, \xi_{x}^{C}$ form a framed $f$-structure $\left(\tilde{J}, \xi_{x}{ }^{V}, \xi_{x}{ }^{C}\right)$ of rank $2 s$ in $T(V)$. Thus we have

PROPOSITION 4.1. If there is given an $s$-frame $\left(\xi_{x}, M\right)$ in $V$, then there exists a framed $f$-structure $\left(\widetilde{J}, \xi_{x} V, \xi_{x}{ }^{C}\right)$ of rank $2 s$ in $T(V)$, where $\widetilde{J}$ is defined by (4.2). If $n=s$, the structure $\widetilde{J}$ is an almost complex structure in $T(V)$.

Let $\xi_{x^{\prime}}$ be other $s$ vector fields such that ${ }^{8)}$

$$
\xi_{y^{\prime}}=a_{y^{\prime}}^{y} \xi_{y},
$$

where the matrix $\left(a_{y^{\prime}}^{y}\right)$ is non-singular. Then for the new $m$-frame $\left(\xi_{y^{\prime}}, M\right)$ the corresponding $s$ covector fields $\eta^{x^{\prime}}$, which satisfy

$$
\eta^{x^{\prime}}\left(\xi_{y^{\prime}}\right)=\delta_{y^{\prime}}^{x^{\prime}}, \quad \eta^{x^{\prime}}(X)=0
$$

for any vector field $X$ belonging to the distribution $M$, are given by

$$
\eta^{x^{\prime}}=a_{x}^{x^{\prime}} \eta^{x},
$$

where the matrix $\left(a_{x}^{x^{\prime}}\right)$ is the inverse of $\left(a_{y^{\prime}}^{y}\right)$. If we put

$$
\widetilde{J}^{\prime}=\xi_{x^{\prime}}, \otimes \eta^{x^{\prime} V}-\xi_{x^{\prime}}{ }^{C} \otimes \eta^{x^{\prime} C},
$$

then we find as a consequence of (4.2)

8) The indices $x^{\prime}, y^{\prime}, z^{\prime}, u^{\prime}, v^{\prime}$ run over the range $\left\{1^{\prime}, 2^{\prime}, \cdots, s^{\prime}\right\}$. 


$$
\begin{array}{r}
\tilde{J}^{\prime}=\widetilde{J}-\left(a_{x^{\prime}}^{z}\right)^{C}\left(a_{y}^{x^{\prime}}\right)^{V} \xi_{z}^{V} \otimes \eta^{y C}-\left(a_{x^{\prime}}^{z}\right)^{V}\left(a_{y}^{x^{\prime}}\right)^{C} \xi_{z}^{C} \otimes \eta^{y V} \\
-\left(a_{x^{\prime}}^{z}\right)^{C}\left(a_{y}^{x^{\prime}}\right)^{C} \xi_{z}{ }^{C} \otimes \eta^{y C},
\end{array}
$$

which implies that the condition

$$
\tilde{J}^{\prime}=\widetilde{J}
$$

is equivalent to the condition that the elements of the matrix $\left(a_{y^{\prime}}^{y}\right)$ are constant. Thus we have

Proposition 4.2. Let there be given in $V$ two s-frames $\left(\xi_{y}, M\right)$ and $\left(\xi_{y^{\prime}}, M\right)$ such that

$$
\xi_{y^{\prime}}=a_{y^{\prime}}^{y} \xi_{y}
$$

the matrix $\left(a_{y^{\prime}}^{y}\right)$ being non-singular. Then the two $f$-structures $\widetilde{J}$ and $\widetilde{J}^{\prime}$ constructed respectively of $\left(\xi_{y}, M\right)$ and $\left(\xi_{y^{\prime}}, M\right)$ following (4.2) coincide with each other in $T(V)$ if and only if the elements of the matrix $\left(a_{y^{\prime}}^{y}\right)$ are constant.

If we take account of (1.16) and (1.17), we get as a consequence of (4.2)

$$
\begin{aligned}
& \tilde{J} X^{V}=-\left(\eta^{x}(X)\right)^{v} \xi_{x}^{c}, \\
& \tilde{J} X^{c}=\left(\eta^{x}(X)\right)^{v} \xi_{x}^{V}-\left(\eta^{x}(X)\right)^{c} \xi_{x}^{c},
\end{aligned}
$$

$X$ being arbitrary vector field in $V$. Especially, we find

$$
\widetilde{J} \xi_{x} V=-\xi_{x}^{C}, \quad \widetilde{J} \xi_{x}{ }^{C}=\xi_{x}{ }^{V}
$$

and

$$
J X^{v}=0, \quad \widetilde{J} X^{c}=0
$$

for any vector field $X$ belonging to the distribution $M$.

The Nijenhuis tensor $\tilde{H}$ of the tensor field $\widetilde{J}$ is by definition

$$
\tilde{H}(\tilde{X}, \tilde{Y})=[\tilde{J} \tilde{X}, \tilde{J} \tilde{Y}]-\tilde{J}[\tilde{J} \tilde{X}, \tilde{Y}]-\tilde{J}[\tilde{X}, \tilde{J} \tilde{Y}]+\tilde{J}^{2}[\tilde{X}, \tilde{Y}]
$$

which reduces to

$$
\tilde{H}(\tilde{X}, \tilde{Y})=[\tilde{J} \tilde{X}, \tilde{J} \tilde{Y}]-\widetilde{J}[\tilde{J} \tilde{X}, \tilde{Y}]-\widetilde{J}[\tilde{X}, \tilde{J} \tilde{Y}]-\left(\xi_{x} \otimes \eta^{x}\right)^{c}[\tilde{X}, \tilde{Y}]
$$

by virtue of (4.3), where $\tilde{X}$ and $\tilde{Y}$ are arbitrary vector fields in $T(V)$. If we take account of (1.14), (1.16), (1.17), (4.6), (4.7) and (4.8), we obtain from (4.9) the following formulas for any vector fields $X$ and $Y$ belonging to the distribution $M$ in $V$ : 
(4. 10)

$$
\begin{aligned}
& \tilde{H}\left(X^{v}, Y^{v}\right)=0 \text {, } \\
& \tilde{H}\left(X^{v}, Y^{c}\right)=-\left(\eta^{x}([X, Y])\right)^{v} \xi_{x}{ }^{v}, \\
& \tilde{H}\left(X^{c}, Y^{c}\right)=-\left(\eta^{x}([X, Y]) \xi_{x}\right)^{c}, \\
& \tilde{H}\left(X^{v}, \xi_{y}^{V}\right)=-\left(\eta^{x}\left(\left[X, \xi_{y}\right]\right)\right)^{v} \xi_{x}^{c}, \\
& \widetilde{H}\left(X^{v}, \xi_{y}^{c}\right)=-\left(\eta^{x}\left(\left[X, \xi_{y}\right]\right) \xi_{x}\right)^{v}, \\
& \tilde{H}\left(X^{c}, \xi_{y}^{V}\right)=-\left(\eta^{x}\left(\left[X, \xi_{y}\right]\right)\right)^{c} \xi_{x}^{c}, \\
& \tilde{H}\left(X^{c}, \xi_{y}^{C}\right)=-\left(\eta^{x}\left(\left[X, \xi_{y}\right]\right)\right)^{c} \xi_{x}{ }^{V}, \\
& \left.\tilde{H}\left(\xi_{y}{ }^{V}, \xi_{z}{ }^{V}\right)=\left[\xi_{y}, \xi_{z}\right]^{c}-2\left(\eta^{x}\left(\left[\xi_{y}, \xi_{z}\right]\right)\right)\right)^{v} \xi_{x}^{c}, \\
& \tilde{H}\left(\xi_{y}{ }^{V}, \xi_{z}{ }^{C}\right)=-\left[\xi_{y}, \xi_{z}\right]^{V}-\left(\eta^{x}\left(\left[\xi_{y}, \xi_{z}\right]\right) \xi_{x}\right)^{V}, \\
& \widetilde{H}\left(\xi_{y}^{C}, \xi_{z}^{C}\right)=\eta^{x}\left(\left[\xi_{y}, \xi_{z}\right]\right)^{V} \xi_{x}^{C}-\left(\eta^{x}\left(\left[\xi_{y}, \xi_{z}\right]\right)\right)^{C} \xi_{x}{ }^{\nu} .
\end{aligned}
$$

Taking account of the integrability condition (3.6), we see that the $f$-structure $J$ is partially integrable if and only if

$$
\widetilde{H}\left(\xi_{y}^{V}, \xi_{z}{ }^{V}\right)=0, \quad \widetilde{H}\left(\xi_{y}{ }^{V}, \xi_{z}{ }^{C}\right)=0, \quad \widetilde{H}\left(\xi_{y}^{c}, \xi_{z}{ }^{c}\right)=0 .
$$

As a direct consequence of (4.10), we see that the condition above is equivalent to the condition

$$
\left[\xi_{y}, \xi_{z}\right]=0
$$

Thus we have

Proposition 4.3. The f-structure $\widetilde{J}$ defined by (4.2) is partially integrable in $T(V)$ if and only if there exist, in each coordinate neighborhood of $V$, local coordinates $\left(x^{h}\right)$ such that

$$
\xi_{1}=\frac{\partial}{\partial x^{1}}, \xi_{2}=\frac{\partial}{\partial x^{2}}, \cdots, \xi_{z}=\frac{\partial}{\partial x^{s}} .
$$

The $f$-structure $\widetilde{J}$ is integrable if and only if we have $\tilde{H}=0$, which is equivalent, by virtue of (4.10), to the conditions

$$
\eta^{x}([X, Y])=0, \quad \eta^{x}\left(\left[X, \xi_{y}\right]\right)=0, \quad\left[\xi_{y}, \xi_{z}\right]=0
$$

for any vector fields $X$ and $Y$ belonging to the distribution $M$. It is easily verified that the conditions (4.12) are equivalent to the conditions

$$
d \eta^{x}=0, \quad\left[\xi_{y}, \xi_{z}\right]=0
$$


which are, by virtue of (4.1), equivalent again to the condition that there exists, in each coordinate neighborhood of $V$, a system of local coordinates $\left(x^{h}\right)$ such that

$$
\begin{aligned}
& \xi_{1}=\frac{\partial}{\partial x^{1}}, \quad \xi_{2}=\frac{\partial}{\partial x^{2}}, \cdots, \quad \xi_{s}=\frac{\partial}{\partial x^{s}} \\
& \eta^{1}=d x^{1}, \quad \eta^{2}=d x^{2}, \quad \cdots, \quad \eta^{s}=d x^{s} .
\end{aligned}
$$

When there exist, in each coordinate neighborhood of $V$, local coordinates $\left(x^{h}\right)$ satisfying (4.14), we say that the given $s$-frame $\left(\xi_{x}, M\right)$ is integrable. Summing up, we have

Proposition 4. 4. The f-stucture $\widetilde{J}$ defined by (4.2) is integrable in $T(V)$ if and only if the given s-frame $\left(\xi_{x}, M\right)$ is integrable in $V$.

Taking account of (1.17), (4.2), (4.6), (4.7) and (4.8), we have from the definition (1.22) of Lie derivative the following formulas for any vector field $X$ in $V$ :

$$
\begin{array}{ll}
\left.\underset{X^{V}}{\mathcal{L}} \widetilde{J}\right) Y^{V}=0, & (\underset{X V}{\mathcal{L}} \tilde{J}) Y^{C}=\left(\eta^{x}([X, Y])\right)^{V} \xi_{x}{ }^{C}, \\
\left.\underset{X^{V}}{(\mathcal{J}} \widetilde{J}\right) \xi_{y}{ }^{V}=-\left[X, \xi_{y}\right]^{V}, & (\underset{X V}{\mathcal{L}} \tilde{J}) \xi_{y}{ }^{C}=\left(\eta^{x}\left(\left[X, \xi_{y}\right]\right)\right)^{V} \xi_{x}{ }^{C},
\end{array}
$$

$Y$ being an arbitrary vector field belonging to the distribution $M$ in $V$. Similarly, we obtain the following formulas for any vector field $X$ in $V$ :

$$
\begin{aligned}
& (\underset{X C}{\mathcal{L}} \tilde{J}) Y^{V}=\left(\eta^{x}([X, Y])\right)^{V} \xi_{x}^{C}, \\
& \underset{X C}{(\mathcal{J}} \widetilde{J}) Y^{C}=\left(\eta^{x}([X, Y])\right)^{V} \xi_{x}^{V}-\left(\eta^{x}([X, Y])\right)^{C} \xi_{x}^{C}, \\
& (\underset{X C}{\mathcal{L}} \widetilde{J}) \xi_{y}^{V}=-\left[X, \xi_{y}\right]^{C}+\left(\eta^{x}\left(\left[X, \xi_{y}\right]\right)\right)^{V} \xi_{x}^{C}, \\
& \underset{X C}{(\mathcal{L}} \widetilde{J}) \xi_{y}^{C}=\left[X, \xi_{y}\right]^{V}-\left(\eta^{x}\left(\left[X, \xi_{y}\right]\right)\right)^{V} \xi_{x}^{V}+\left(\eta^{x}\left(\left[X, \xi_{y}\right]\right)\right)^{C} \xi_{x}^{C},
\end{aligned}
$$

$Y$ being an arbitrary vector field belonging to the distribution $M$ in $V$.

According to the formula (4.15), the condition $\underset{X V}{\mathcal{L}} \widetilde{J}=0$ is equivalent to the conditions $\left[X, \xi_{y}\right]=0, \eta^{x}([X, Y])=0$ for any vector field $Y$ belonging to $M$, which are equivalent again to the conditions

$$
\mathcal{L}_{X} \xi_{y}=0, \quad \underset{X}{\mathcal{L}} \eta^{x}=0 .
$$

According to the formula (4.16), the condition $\underset{x^{C}}{\mathcal{L}} \widetilde{J}=0$ is equivalent to the conditions

$$
\left[X, \xi_{y}\right]=C_{y}{ }^{x} \xi_{x}, \quad \eta^{x}([X, Y])=0
$$

for any vector field $Y$ belonging to $M$, where $C_{y}{ }^{x}$ are constant. This condition is 
equivalent to the conditions

$$
\mathcal{L}_{X} \xi_{y}=C_{y}^{x} \xi_{x}, \quad \mathcal{L}_{X} \eta^{x}=-C_{y}{ }^{x} \eta^{y},
$$

$C_{y}{ }^{x}$ being constant. Summing up, we have

Proposition 4.5. Let there be given an s-frame $\left(\xi_{x}, M\right)$ in $V$. Then the vertical lift $X^{V}$ of a vector field $X$ given in $V$ satisfies the condition

$$
\underset{X V}{\mathcal{L}} \widetilde{J}=0
$$

in $T(V)$ if and only if the conditions

$$
\mathcal{L}_{X} \xi_{y}=0, \quad \mathcal{L}_{X} \eta^{x}=0
$$

are satisfied in $V$. The complete lift $X^{c}$ of a vector field $X$ given in $V$ satisfies the condition

$$
\underset{X^{C}}{\mathcal{L}} \tilde{J}=0
$$

in $T(V)$ if and only if the conditions

$$
\mathcal{L}_{X} \xi_{y}=C_{y}{ }^{x} \xi_{x}, \quad \mathcal{L}_{X} \eta^{x}=C_{y}{ }^{x} \eta^{y}
$$

are satisfied with constants $C_{y}{ }^{x}$ in $V$, where $\tilde{J}$ is defined by (4.2).

As is well known, there exists in a Lie group $G n$ left invariant vector fields $\xi_{1}, \xi_{2}, \cdots, \xi_{n}$, which form a basis of the Lie algebra of $G$, if $\operatorname{dim} G=n$. Thus, according to Proposition 4.1, there exists, in the tangent bundle $T(G)$ of $G$, an almost complex structure $\tilde{J}$ corresponding to the $n$-frame $\left(\xi_{x}\right)(x=1,2, \cdots, n)$. The almost complex structure $\widetilde{J}$ thus introduced in $T(G)$ does not depend on the choice of the base $\left(\xi_{x}\right)$ of the Lie algebra of $G$ because of Proposition 4.2. Taking account of Proposition 4. 4, we see that the almost complex structure $\widetilde{J}$ is complex analytic in $T(G)$ if and only if the Lie group $G$ is abelian. Thus we have

PROPOSITION 4.6. There exists canonically an almost complex structure $\tilde{J}$ in the tangent bundle $T(G)$ of any Lie group $G$. The almost complex structure $\widetilde{J}$ is complex analytic if and only if the Lie group $G$ is abelian.

According to Proposition 4.5, we have directly

Proposition 4.7. For any left invariant vector field $X$ given in a Lie group $G$, its complete lift $X^{C}$ is almost analytic in $T(G)$ with respect to the almost complex structure $\tilde{J}$ mentioned in Proposition 4.6. For a left invariant vector field $X$ given in $G$, its vertical lift $X^{V}$ is almost analytic in $T(G)$ with respect to the almost complex structure $\widetilde{J}$ if and only if $X$ belongs to the center of the Lie 
algebra of $G$.

As a corollary to Proposition 4.7 , we have

Proposition 4.8. Let $G$ be an abelian Lie group. Then, for any left invariant vector field $X$ in $G$, its vertical lift $X^{v}$ and its complete lift $X^{c}$ are analytic in $T(G)$ with respect to the complex structure $\tilde{J}$ mentioned in Proposition 4.6. The tangent bundle $T(G)$ of any abelian Lie group $G$ is a homogeneous complex space.

Given an element $\alpha$ of a Lie group $G$, we denote by $L(\alpha)$ and $R(\alpha)$ respectively the left and the right translations of $G$, which correspond to the element $\alpha$ of $G$. Take a base $\left(\xi_{x}\right)$ of the Lie algebra of $G$. The $n$ vector fields $\xi_{x}$ being left invariant, we have

$$
\begin{aligned}
& L(\alpha) \xi_{x}=\xi_{x}, \\
& R(\alpha) \xi_{x}=C_{x}^{y}(\alpha) \xi_{y}
\end{aligned} \quad(x, y=1,2, \cdots, n)
$$

for any element $\alpha$ of $G$, where $C_{y}{ }^{x}(\alpha)$ are constants determined by $\alpha$. Thus, taking account of Proposition 4.2 and following notations given in $\S 1$, we have

Proposition 4.9. Let $G$ be a Lie group. Then, for any element $\alpha$ of $G$, the corresponding transformations $L(\alpha)^{C}$ and $R(\alpha)^{C}$ of $T(T(G)$ ) leave invariant the almost complex structure $\widetilde{J}$ mentioned in Proposition 4.6.

\section{§5. Almost contact structures and framed $f$-structures.}

Let there be given, in an $n$-dimensional differentiable manifold $V$, a tensor field $f$ of type (1.1), a vector field $\xi$ and a covector field $\eta$ satisfying

$$
\begin{array}{ll}
f^{2}=-I+\xi \otimes \eta, & f \xi=0, \\
\eta(f X)=0, & \eta(\xi)=1
\end{array}
$$

for any vector field $X$ in $V$. Then $n$ is necessarily odd. We call a structure defined by the set $(f, \xi, \eta)$ of such tensor fields $f, \xi$ and $\eta$ an almost contact structure (c.f. Sasaki [6]). Taking account of (5.1), we see easily that

$$
f^{3}+f=0
$$

and that $f$ is of rank $n-1$ everywhere in $V$. Thus the set $(f, \xi)$ is a framed $f$ structure in $V$. Conversely, if there is given an $f$-structure $f$ of rank $n-1$ in an $n$-dimensional differentiable manifold $V, V$ being assumed to be orientable, then there exists an almost contact structure $(f, \xi, \eta)$ in $V$. When the framed $f$ structure $(f, \xi)$ is normal, the given almost contact structure $(f, \xi, \eta)$ is said to be 
normal (c.f. Sasaki and Hatakeyama [7]).

If there is given an almost contact structure $(f, \xi, \eta)$ in $V$, then the tensor field $S(X, Y)$ constructed by (3.9) from the framed $f$-structure $(f, \xi)$ reduces to

$$
S(X, Y)=N(X, Y)+(X \eta(Y)-Y \eta(X)-\eta([X, Y])) \xi
$$

for any vector fields $X$ and $Y$ in $V$, where $N$ denotes the Nijenhuis tensor of $f$ (c.f. Sasaki and Hatakeyama [7]). The almost contact structure $(f, \xi, \eta)$ is normal if and only if we have $S=0$ (c.f. Sasaki and Hatakeyama [7]).

If we introduce a tensor field $S_{1}$ of type $(0,2)$, a tensor field $S_{2}$ of type $(1,1)$ and a covector field $S_{3}$ by the formulas

$$
\begin{aligned}
& S_{1}(X, Y)=(f X) \eta(Y)-(f Y) \eta(X)-\eta([f X, Y]+[X, f Y]) \\
& =\left(\mathcal{f}_{f X} \eta\right) Y-\left(\mathcal{f}_{f Y} \eta\right) X, \\
& S_{2}(X)=[\xi, f X]-f[\xi, X] \\
& =(\underset{\xi}{\mathcal{L}} f) X, \\
& S_{3}(X)=(\xi(\eta(X))-\eta([\xi, X]) \\
& =\left(\mathcal{L}_{\xi} \eta\right) X
\end{aligned}
$$

respectively, $X$ and $Y$ being arbitrary vector fields in $V$, then we see the well known fact that $S_{1}, S_{2}$ and $S_{3}$ vanish identically in $V$ if $S$ vanishes identically in $V$ (Sasaki and Hatakeyama [7]).

We find directly from (5.2) and (5.3) the following formulas:

$$
\begin{aligned}
S(X, Y) & =[f X, f Y]-f[f X, Y]-f[X, f Y]-[X, Y], \\
S_{1}(X, Y) & =-\eta([f X, Y]+[X, f Y]), \\
S_{2}(X) & =[\xi, f X]-f[\xi, X], \\
S_{3}(X) & =-\eta([\xi, X]),
\end{aligned}
$$

$X$ and $Y$ being arbitrary vector fields satisfying $\eta(X)=0$ and $\eta(Y)=0$ in $V$;

$$
\begin{aligned}
& S(X, \xi)=f[\xi, f X]+[\xi, X], \\
& S_{1}(X, \xi)=\eta([\xi, f X]),
\end{aligned}
$$

$X$ being an arbitrary vector field satisfying $\eta(X)=0$ in $V$, and

$$
S_{2}(\xi)=0, \quad S_{3}(\xi)=0 .
$$

Taking account of (1.14), (1.16), (1.18) and (1.19), we find from (5.1) 


$$
\begin{aligned}
& \left(f^{C}\right)^{2}=-I+\xi^{V} \otimes \eta^{C}+\xi^{C} \otimes \eta^{V}, \\
& f^{C} \xi^{V}=0, \quad f^{C} \xi^{C}=0, \quad \eta^{V}\left(f^{C} \tilde{X}\right)=0, \quad \eta^{C}\left(f^{C} \tilde{X}\right)=0, \\
& \eta^{V}\left(\xi^{V}\right)=0, \quad \eta^{V}\left(\xi^{C}\right)=1, \quad \eta^{C}\left(\xi^{V}\right) \quad=1, \quad \eta^{C}\left(\xi^{C}\right) \quad=0
\end{aligned}
$$

for any vector field $\tilde{X}$ in $T(V)$.

If we now define a tensor field $\widetilde{J}$ of type $(1,1)$ in $T(V)$ by

$$
\widetilde{J}=f^{C}+\xi^{V} \otimes \eta^{V}-\xi^{C} \otimes \eta^{C},
$$

then we find

$$
\widetilde{J}^{2}=-I
$$

as a direct consequence of (5.7) and (5.8). Therefore the tensor field $\tilde{J}$ defined by (5.8) is an almost complex structure in $T(V)$. The almost complex structure $\widetilde{J}$ has components of the form

$$
\left(\begin{array}{lc}
f_{j}^{h}-\left(\partial \eta_{j}\right) \xi^{h} & -\eta_{j} \xi^{h} \\
\partial f_{j}^{h}-\left(\partial \eta_{j}\right)\left(\partial \xi^{h}\right)+\eta_{j} \xi^{h} & f_{j}^{h}-\eta_{j} \partial \xi^{h}
\end{array}\right)
$$

with respect to the induced local coordinates $\left(x^{h}, y^{h}\right)$ in $\pi^{-1}(U) \subset T(V)$, where $f_{j}{ }^{h}$, $\xi^{h}$ and $\eta_{j}$ denote respectively the components of $f, \xi$ and $\eta$ with respect to local coordinates $\left(x^{h}\right)$ defined in a coordinate neighborhood $U$ of $V$ (c.f. $\S 1$ ). Thus we have

Proposition 5. 1. There exists in $T(V)$ an almost complex structure $\tilde{J}$ defined by (5. 8), if there is given an almost contact structure $(f, \xi, \eta)$ in $V$. (Tanno [9], [10])

Taking account of (1.16) and (1.18), we get from (5.8) the following formulas:

$$
\begin{aligned}
& \widetilde{J} X^{v}=(f X)^{V}-(\eta(X))^{v} \xi^{C}, \\
& \widetilde{J} X^{C}=(f X)^{C}+(\eta(X))^{v} \xi^{V}-(\eta(X))^{c} \xi^{C},
\end{aligned}
$$

$X$ being an arbitrary vector field in $V$. Especially, we find

$$
\begin{array}{ll}
\widetilde{J} X^{V}=(f X)^{V}, & \widetilde{J} X^{C}=(f X)^{C}, \\
\widetilde{J} \xi^{V}=-\xi^{C}, & \widetilde{J} \xi^{C}=\xi^{V},
\end{array}
$$

$X$ being an arbitrary vector field satisfying $\eta(X)=0$ in $V$.

The Nijenhuis tensor $\widetilde{H}$ of the almost complex structure $\widetilde{J}$ is by definition

$$
\tilde{H}(\tilde{X}, \tilde{Y})=[\tilde{J} \tilde{X}, \tilde{J} \tilde{Y}]-\tilde{J}[\tilde{J} \tilde{X}, \tilde{Y}]-\tilde{J}[\tilde{X}, \tilde{J} \tilde{Y}]-[\tilde{X}, \tilde{Y}]
$$


for any vector fields $\tilde{X}$ and $\tilde{Y}$ in $T(V)$. Thus, taking account of (1.17), (1. 18), (5.4), (5.5), (5.7), (5.8) and (5.11), we obtain the following formulas for any vector fields $X$ and $Y$ satisfying $\eta(X)=0$ and $\eta(Y)=0$ in $V$ :

$$
\begin{aligned}
& \tilde{H}\left(X^{v}, Y^{v}\right)=0 \text {, } \\
& \tilde{H}\left(X^{v}, Y^{v}\right)=(S(X, Y))^{V}-\left(S_{1}(X, Y)\right)^{V} \xi^{C}, \\
& \tilde{H}\left(X^{c}, Y^{c}\right)=(S(X, Y))^{C}+\left(S_{1}(X, Y)\right)^{V} \xi^{V}-\left(S_{1}(X, Y)\right)^{C} \xi^{C}, \\
& \tilde{H}\left(X^{v}, \xi^{v}\right)=\left(S_{2}(X)\right)^{v}+\left(S_{8}(X)\right)^{v} \xi^{C}, \\
& \tilde{H}\left(X^{v}, \xi^{C}\right)=\left(S_{2}(X)\right)^{C}+(S(X, \xi))^{V}+\left(S_{3}(X)\right)^{v} \xi^{V}-\left(S_{1}(X, \xi)\right)^{V}+\left(S_{3}(X)\right)^{c} \xi^{C}, \\
& \tilde{H}\left(X^{c}, \xi^{V}\right)=(S(X, \xi))^{V}-\left(S_{1}(X, \xi)\right)^{v} \xi^{C}, \\
& \left.\widetilde{H}\left(X^{C}, \xi^{C}\right)=(S(X, \xi))^{C}-\left(S_{2}(X)\right)^{V}+S_{1}(X, \xi)\right)^{V} \xi^{V}-\left\{\left(S_{1}(X, \xi)\right)^{C}-\left(S_{3}(X)\right)^{V}\right\} \xi^{C}, \\
& \widetilde{H}\left(\xi^{V}, \xi^{C}\right)=0 .
\end{aligned}
$$

As a direct consequence of $(5.12)$, we see that the condition $\tilde{H}=0$ is equivalent to the conditions

$$
S(X, Y)=0, \quad S_{1}(X, Y)=0, \quad S_{2}(X)=0, \quad S_{3}(X)=0,
$$

$$
S(X, \xi)=0, \quad S_{1}(X, \xi)=0
$$

for any vector fields $X$ and $Y$ satisfying $\eta(X)=0$ and $\eta(Y)=0$ in $V$. However, we have $S(\xi, \xi)=0$ and $S_{1}(\xi, \xi)=0$ because of the skew-symmetry of $S$ and $S_{1}$. Thus, taking account of $(5.6)$, we see that the conditions $(5.13)$ are equivalent to the conditions

$$
S=0, \quad S_{1}=0, \quad S_{2}=0, \quad S_{3}=0,
$$

which are again equivalent to the condition

$$
S=0 \text {, }
$$

because $S=0$ implies $S_{1}=0, S_{2}=0, S_{3}=0$. Summing up, we have

Proposition 5.2. The almost complex structure $\widetilde{J}$ defined by (5.8) is complex analytic in $T(V)$ if and only if the given almost contact structure $(f, \xi, \eta)$ is normal in $V$. (Tanno [10])

Let there be given an almost contact structure $(f, \xi, \eta)$ in $V$. Then the tensor fields $f^{C}, \xi^{V}, \xi^{C}$. form in $T(V)$ a framed $f$-structure $\left(f^{C}, \xi^{V}, \xi^{C}\right)$ of rank $2 n-2$, if $\operatorname{dim} V=n$. Thus, taking account of Proposition 3.3, we have 
Proposition 5. 3. Let $(f, \xi, \eta)$ be an almost contact structure in $V$. Then the framed $f$-structure $\left(f^{C}, \xi^{V}, \xi^{C}\right)$ is normal in $T(V)$ if and only if the given almost contact structure $(f, \xi, \eta)$ is normal in $V$.

Propositions 5.2 and 5.3 imply immediately that the following two conditions (a) and (b) are equivalent to each other:

(a) The almost complex structure $\widetilde{J}$ in $T(V)$ defined by (5.8) is complex analytic;

(b) The framed $f$-structure $\left(f^{C}, \xi^{V}, \xi^{C}\right)$ is normal in $T(V)$, when there is given an almost contact structure $(f, \xi, \eta)$ in $V$.

Given an almost contact structure $(f, \xi, \eta)$ in $V$, a necessary and sufficient condition for the $f$-structure $f$ in $V$ to be integrable is that there exist in each coordinate neighborhood of $V$ local coordinates with respect to which the tensor fields $f, \xi$ and $\eta$ have respectively components of the form

$$
\left(f_{j}{ }^{h}\right)=\left(\begin{array}{ccc}
0 & -E_{m} & 0 \\
E_{m} & 0 & 0 \\
0 & 0 & 0
\end{array}\right), \quad\left(\xi^{h}\right)=\left(\begin{array}{c}
0 \\
\vdots \\
0 \\
c
\end{array}\right), \quad\left(\eta_{j}\right)=\left(0, \cdots, 0, c^{-1}\right),
$$

$c$ being a certain function in $V$ and $E_{m}$ denoting the unit $m \times m$ matrix, where $m=(n-1) / 2, n=\operatorname{dim} V$. On the other hand, we see, by means of Proposition 3. 3, that a necessary and sufficient condition for the $f$-structure $f^{C}$ to be integrable in $T(V)$ is that the $f$-structure $f$ is integrable in $V$. Thus we have

Proposition 5. 4. Let $(f, \xi, \eta)$ be an almost contact structure in $V$. Then the $f$-structure $f^{c}$ in $T(V)$ is integrable if and only if the given tensor fields $f, \xi$ and $\eta$ have respectively components given in (5.14) with respect to a suitable local coordinates in each coordinate neighborhood of $V$.

Let $\mu: V \rightarrow V$ be a differentiable transformation of an $n$-dimensional manifold $V$ with an almost contact structure $(f, \xi, \eta)$. Then, taking account of (1.29) and (1. 30), we obtain

$$
\mu^{C} \hat{J}=(\mu f)^{C}+(\mu \xi)^{V} \otimes(\mu \eta)^{V}-(\mu \xi)^{C} \otimes(\mu \eta)^{C}
$$

where $\tilde{J}$ is the almost complex structure defined in $T(V)$ by (5.8). Denoting by $\bar{f}_{j}{ }^{h}, \bar{\xi}^{h}$ and $\bar{\eta}_{j}$ the components of $\mu f, \mu \xi$ and $\mu \eta$ respectively and taking account of (5.9), we see that the condition

$$
\mu^{c} \widetilde{J}=J
$$

is expressed by 


$$
\left(\begin{array}{lc}
\bar{f}_{j}^{h}-\left(\partial \bar{\eta}_{j}\right) \bar{\xi}^{h} & -\bar{\eta}_{j} \bar{\xi}^{h} \\
\partial \bar{f}_{j}^{h}-\left(\partial \bar{\eta}_{j}\right)\left(\partial \bar{\xi}^{h}\right)+\bar{\eta}_{j} \bar{\xi}^{h} & \bar{f}_{\jmath}^{h}-\bar{\eta}_{j}\left(\partial \bar{\xi}^{h}\right)
\end{array}\right)
$$

$$
=\left(\begin{array}{lc}
f_{j}^{h}-\left(\partial \eta_{j}\right) \xi^{h} & -\eta_{j} \xi^{h} \\
\partial f_{j}^{h}-\left(\partial \eta_{j}\right)\left(\partial \xi^{h}\right)+\eta_{j} \xi^{h} & f_{j}^{h}-\eta_{j}\left(\partial \xi^{h}\right)
\end{array}\right)
$$

with respect to induced local coordinates $\left(x^{h}, y^{h}\right)$ in $T(V)$, where $f_{j}^{h}, \xi^{h}$ and $\eta_{j}$ are the components of $f, \xi$ and $\eta$ respectively. From (5.17) it follows

$$
\bar{\eta}_{j} \bar{\xi}^{h}=\eta_{j} \xi^{h},
$$

which implies together with $\eta(\xi)=1$ and $(\mu \eta)(\mu \xi)=1$ that

$$
\bar{\xi}^{h}=a \xi^{h}, \quad \bar{\eta}_{J}=a^{-1} \eta_{\jmath}
$$

hold with a non-zero function $a$. From (5.17) we get

$$
\bar{f}_{j}{ }^{h}-\left(\partial \bar{\eta}_{j}\right) \bar{\xi}^{h}=f_{j}^{h}-\left(\partial \eta_{j}\right) \xi^{h}, \quad \bar{f}_{j}^{h}-\bar{\eta}_{j}\left(\partial \bar{\xi}^{h}\right)=f_{j}^{h}-\eta_{j}\left(\partial \xi^{h}\right),
$$

which imply together with $(5.18)$

$$
\bar{f}_{j}^{h}+\alpha\left(\partial \alpha^{-1}\right) \eta_{j} \xi^{h}=f_{j}^{h}, \quad \bar{f}_{j}{ }^{h}+\alpha^{-1}(\partial \alpha) \eta_{j} \xi^{h}=f_{j}^{h} .
$$

Substracting these two equations, we obtain

$$
a\left(\partial a^{-1}\right)=a^{-1}(\partial a)
$$

which implies $\partial a=0$. That is, the function $a$ is necessarily a constant $c$. Therefore, we find from (5.18) and (5.19)

$$
\bar{f}_{j}^{h}=f_{j}^{h}, \quad \bar{\xi}^{h}=c \xi^{h}, \quad \bar{\eta}_{\jmath}=c^{-1} \eta_{\jmath},
$$

i.e.

$$
\bar{f}=f, \quad \bar{\xi}=c \xi, \quad \bar{\eta}=c^{-1} \eta
$$

with a constant $c$. Summing up we have

Proposition 5.5. Let $\mu: V \rightarrow V$ be a differentiable transformation of a differentiable manifold $V$ with an almost contact structure $(f, \xi, \eta)$. The differential map $\mu: T(V) \rightarrow T(V)$ preserves the almost complex structure $\widetilde{J}$ defined by (5.8) if and only if the conditions 


$$
\mu f=f, \quad \mu \xi=c \xi, \quad \mu \eta=c^{-1} \eta
$$

are satisfied with a non-zero constant $c$ in $V$. (Tanno [10])

Let $X$ be a vector field in $V$. Then, taking account of the definition (1.22) of Lie derivative and (5.8), we find the following formulas for any vector field $X$ in $V$ :

$$
\begin{aligned}
& (\underset{X V}{\mathcal{L}} \widetilde{J}) Y^{V}=0, \\
& (\underset{X V}{\mathcal{L}} \tilde{J}) Y^{C}=\left(\left(\mathcal{L}_{X} f\right) Y\right)^{V}-\left(\left(\mathcal{L}_{X} \eta\right)(Y)\right)^{V} \xi^{C}, \\
& \underset{X V}{(\mathcal{L}} \widetilde{J}) \xi^{V}=-\left(\mathcal{L}_{X} \xi\right)^{V}, \\
& \underset{X V}{(\mathcal{L}} \widetilde{J}) \xi^{C}=\left(\left(\mathcal{X}_{X} f\right) \xi\right)^{V}-\left(\left({\underset{X}{\mathcal{L}}}_{\mathcal{L}} \eta\right)(\xi)\right)^{V} \xi^{C},
\end{aligned}
$$

$\widetilde{J}$ being defined by (5.8) and $Y$ being an arbitrary vector field satisfying $\eta(Y)=0$ in $V$. By a similar device, we get the following formulas for any vector field $X$ in $V$ :

$$
\begin{aligned}
& \left(\underset{X^{C}}{\mathcal{L}} \tilde{J}\right) Y^{V}=\left(\left(\mathcal{L}_{X} f\right) Y\right)^{V}-\left(\left(\mathcal{L}_{\boldsymbol{X}} \eta\right)(Y)\right)^{V} \xi^{C}, \\
& \left(\underset{X^{C}}{\mathcal{L}} \widetilde{J}\right) Y^{C}=\left(\left(\mathcal{L}_{\boldsymbol{X}} f\right) Y\right)^{C}+\left(\left(\mathcal{L}_{X} \eta\right)(Y)\right)^{V} \xi^{V}-((\mathcal{X} \eta)(Y))^{C} \xi^{C}, \\
& (\underset{X C}{\mathcal{L}} \widetilde{J}) \xi^{V}=((\underset{X}{\mathcal{L}} f) \xi)^{V}-[X, \xi]^{C}-\left(\left({\underset{X}{X}}_{\mathcal{L}} \eta\right)(\xi)\right)^{V} \xi^{C}, \\
& \underset{X C}{(\mathcal{L}} \tilde{J}) \xi^{C}=\left(\left(\mathcal{L}_{X} f\right) \xi\right)^{C}+[X, \xi]^{V}+\left(\left({\underset{X}{X}}_{\mathcal{L}} \eta\right)(\xi)\right)^{V} \xi^{V}-((\mathcal{X} \eta)(\xi))^{C} \xi^{C},
\end{aligned}
$$

$\widetilde{J}$ being defined by (5.8) and $Y$ being an arbitrary vector field satisfying $\eta(Y)=0$ in $V$.

As a consequence of (5.20), we see that the condition

$$
\underset{X V}{\mathcal{L}} \widetilde{J}=0
$$

is equivalent to the conditions

$$
\underset{X}{\mathcal{L} f}=0, \quad \mathcal{L}_{\boldsymbol{X}} \xi=0, \quad \mathcal{L}_{X} \eta=0 .
$$

From (5.21) it follows that the condition

$$
\underset{x C}{\mathcal{L}} \tilde{J}=0
$$


is equivalent to the conditions

$$
\mathcal{L}_{X} f=0, \quad \mathcal{L}_{X} \xi=c \xi, \quad \mathcal{L}_{X} \eta=-c \eta,
$$

$c$ being a constant. Thus we have

Proposition 5.6. Let there be given an almost contact structure $(f, \xi, \eta)$ in $V$. Then the vertical lift $X^{V}$ of a vector field $X$ given in $V$ is almost analytic with respect to the almost complex structure $\widetilde{J}$ in $T(V), \widetilde{J}$ being the almost complex structure defined by (5.8), if and only if the conditions

$$
\mathcal{L}_{X} f=0, \quad \mathcal{L}_{X} \xi=0, \quad \mathcal{L}_{X} \eta=0
$$

are satisfied in $V$. (Tanno [10])

Proposition 5.7. Under the same assumption as in Proposition 5.6, the complete lift $X^{C}$ of a vector field $X$ is almost analytic with respect to the almost complex structure $\widetilde{J}$ in $T(V)$, if and only if the conditions

$$
\mathcal{L}_{X} f=0, \quad \mathcal{L}_{X} \xi=c \xi, \quad \mathcal{L}_{X} \eta=-c \eta
$$

are satisfied with a constant $c$ in $V$. (Tanno [10])

Proposition 5.7 is a direct consequence of Proposition 5. 5.

Let there be given a framed $f$-structure $\left(f, \xi_{x}\right)$ of rank $r$ in an $n$-dimensional differentiable manifold $V$ (c.f. $\S 3$ ). Then, taking account of the fact $m=\xi_{x} \otimes \eta^{x}$, we find from (3.2) and (3.10)

$$
\begin{gathered}
f^{2}=-I+\xi_{x} \otimes \eta^{x}, \\
\eta^{x}(f X)=0, \quad \eta^{x}\left(\xi_{y}\right)=\delta_{y}^{x}
\end{gathered}
$$

for any vector field $X$ in $V .^{9)}$ If we put $s=n-r$ and define $s$ tensor fields $S_{(1)}{ }^{x}$ of type $(0,2), s$ tensor fields $S_{(2) y}$ of type $(1,1), s^{2}$ covector fields $S_{(3) y}{ }^{x}$ and $s(s-1) / 2$ vector fields $S_{(4) y z}$ respectively by the following formulas:

$$
\begin{aligned}
S_{(1)}^{x}(X, Y) & =(f X) \eta^{x}(Y)-(f Y) \eta^{x}(X)-\eta^{x}([f X, Y]+[X, f Y]), \\
S_{(2) y}(X) & =\left[\xi_{y}, f X\right]-f\left[\xi_{y}, X\right], \\
S_{(3) y}{ }^{x}(X) & =\xi_{y}\left(\eta^{x}(X)\right)-\eta^{x}\left(\left[\xi_{y}, X\right]\right), \\
S_{(4) y z} & =\left[\xi_{y}, \xi_{z}\right],
\end{aligned}
$$

9) The indices $x, y, z, u, v$ run over the range $\{1,2, \cdots, s\}$, where $s=n-r$. 
where $X$ and $Y$ are arbitrary vector fields in $V$, then we see that the tensor fields $S_{(1)}^{x}, S_{(2) y}, S_{(3) y} x, S_{(4) y z}$ vanish identically in $V$ if the tensor field $S$ defined by (3.9) vanishes identically in $V$ (c.f. Ishihara [1], Nakagawa [4]).

If we now define a tensor field $\widetilde{J}$ of type $(1,1)$ in $V$ by

$$
\widetilde{J}=f^{C}+\xi_{x} V \otimes \eta^{x V}-\xi_{x}^{C} \otimes \eta^{x C}
$$

then we find

$$
\widetilde{J}^{2}=-I
$$

by making use of the formulas

$$
\begin{aligned}
& \left(f^{C}\right)^{2}=-I+\xi_{x}{ }^{V} \otimes \eta^{x} C+\xi_{x}{ }^{C} \otimes \eta^{x} V \\
& f^{C} \xi_{y}{ }^{V}=0, \quad \quad f^{C} \xi_{y}{ }^{C}=0, \\
& \eta^{x V}\left(f^{c} \tilde{X}\right)=0, \quad \eta^{x C}\left(f^{c} \tilde{X}\right)=0, \\
& \eta^{x}\left(\xi_{y}{ }^{V}\right)=0, \quad \quad \eta^{x}\left(\xi_{y}^{C}\right)=\delta_{y}^{x}, \\
& \eta^{x C}\left(\xi_{y}{ }^{V}\right)=\delta_{y}^{x} \quad \eta^{x C}\left(\xi_{y}{ }^{C}\right)=0,
\end{aligned}
$$

which are direct consequences of (5.24), where $\tilde{X}$ is an arbitrary vector field in $T(V)$. Therefore the tensor field $\widetilde{J}$ defined by $(5.26)$ is an almost complex structure in $T(V)$. Thus we have

Proposition 5. 8. There exists in $T(V)$ an almost complex structure $\tilde{J}$ defined by (5.26) if there is given a framed $f$-structure $\left(f, \xi_{x}\right)$ in $V$.

Denoting by $\tilde{H}$ the Nijenhuis tensor of the almost complex structure $\tilde{J}$ defined by (5.26), we have, by a similar device as that used to get (5.12), formulas expressing $\tilde{H}$ in terms of tensor fields $S, S_{(1)}^{x}, S_{(2) y}, S_{(3) y}{ }^{x}, S_{(4) y z}$. Consequently, we have

Proposition 5.9. The almost complex structure $\tilde{J}$ in $T(V)$ defined by (5.26) is complex analytic if and only if the given framed $f$-structure $\left(f, \xi_{x}\right)$ is normal in $V$.

By a similar method as that used to get Propositions 5.5, 5.6 and 5.7, we have respectively

Proposition 5.10. Let $\mu: V \rightarrow V$ be a differentiable transformation of $V$ with a framed $f$-structure $\left(f, \xi_{x}\right)$. The differential map $\mu: T(V) \rightarrow T(V)$ preserves the almost complex structure $\widetilde{J}$ defined by (5.26) if and only if the conditions 


$$
\mu f=f, \quad \mu \xi_{y}=C_{y}{ }^{x} \xi_{x}, \quad \mu \eta \eta^{x}=B_{y}{ }^{x} \eta^{y}
$$

are satisfied in $V$, where the matrix $\left(C_{y}^{x}\right)$ is non-singular and constant and the matrix $\left(B_{y}^{x}\right)$ is the inverse of $\left(C_{y}^{x}\right)$.

Proposition 5.11. Let there be given a framed $f$-structure $\left(f, \xi_{x}\right)$ in $V$. Then the vertical lift $X^{V}$ of a vector field $X$ given in $V$ is almost analytic with respect to the almost complex structure $\widetilde{J}$ defined by $(5.26)$ in $T(V)$ if and only if the conditions

$$
\mathcal{L}_{X} f=0, \quad \mathcal{L}_{X} \xi_{y}=0, \quad \mathcal{L}_{X} \eta^{x}=0
$$

are satisfied in $V$.

Proposition 5.12. Under the same assumptions as in Proposition 5.11, the complete lift $X^{C}$ of a vector field $X$ given in $V$ is almost analytic with respect to the almost complex structure $\widetilde{J}$ in $T(V)$ if and only if the conditions

$$
\mathcal{L}_{X} f=0, \quad \mathcal{L}_{X} \xi_{y}=C_{y}{ }^{x} \xi_{x}, \quad \mathcal{L}_{X} \eta^{x}=-C_{y}{ }^{x} \eta^{y}
$$

are satisfied with constants $C_{y}^{x}$ in $V$.

Proposition 5.12 is a corollary to Proposition 5.10. Propositions 5.1, 5.2, 5.5, 5.6 and 5.7 are respectively obtained as corollaries to Propositions 5.8, 5.9, 5.10, 5.11 and 5.12 .

\section{REFERENCES}

[1] Ishinara, S., Normal structure $f$ satısfying $f^{3}+f=0$. Kōdal Math. Sem. Rep. 18 (1966), 36-47.

[2] IshinaRA, S., AND K. YANO, On integrability of a structure $f$ satisfying $f^{3}+f=0$. Quart. J. Math. Oxford (2) 15 (1964), 217-222.

[3] LEDGer, A. J., AND K. YANO, The tangent bundle of a locally symmetric space. J. of London Math. Soc. 40 (1965), 487-492.

[4] Nakagawa, H., f-structure induced on submanifolds in space, almost Hermitian or Kählerıan. Kōda1 Math. Sem. Rep. 18 (1966), 161-183.

[5] SASAKI, S., On the differential geometry of tangent bundles of Riemannian manifolds, Tôhoku Math. J. 10 (1958), 7-15.

[6] SASAKI, S., On differentiable manifolds with certain structures which are closely related to almost contact structure I. Tôhoku Math. J. 12 (1960), 459-476.

[7] SASAKI, S., AND Y. HATAKEYAMA, On differentiable manifolds with certain structures which are closely related to almost contact structure II. Tôhoku Math. J. 13 (1961), 281-294.

[8] SATō, I., Almost analytıc vector fields in almost complex manifolds. Tôhoku Math. J. 17 (1965), 185-199.

[9] TANNo, S., Almost complex structures in bundle spaces over almost contact 
manifolds. J. of Math. Soc. Japan 17 (1965), 167-186.

[10] Tanno, S., An almost complex structure of the tangent bundle of an almost contact manifold. Tôhoku Math. J. 17 (1965), 7-15.

[11] Yano, K., On a structure $f$ satısfying $f^{3}+f=0$, Technical Report No. 12 (1961), Univ. of Washington.

[12] YANO, K., On a structure defined by a tensor field $f$ of type $(1,1)$ satisfying $f^{3}+f=0$. Tensor, N. S. 14 (1963), 99-109.

[13] Yano, K., AND S. Kobayashi, Prolongation of tensor fields and connections to tangent bundles, I. General theory. J. of Math. Soc. Japan 18 (1966), 194-210.

[14] Yano, K., AND S. Kobayashi, Prolongation of tensor fields and connections to tangent bundles, II. Affine automorphisms. J. of Math. Soc. Japan 18 (1966), 236-246.

[15] YANO, K., AND A. J. LedGeR, Linear connections on tangent bundles. J. of London Math. Soc. 39 (1964), 495-500.

Department of Mathematics,

Tokyo Institute of Technology. 